\title{
Robust quadri-phase audio watermarking
}

\author{
Fei Yan, Bing Ji, De Zhang and Hui Fang \\ Institute of Acoustics, State Key Lab of Modern Acoustics, Nanjing University, \\ Nanjing 210093, P.R. China
}

(Received 29 April 2003, Accepted for publication 12 June 2003 )

Keywords: Robust, Quadri-phase sequence, DWT

PACS number: 43.60.-c [DOI: 10.1250/ast.25.106]

\section{Introduction}

In the past decade, there has been an explosion in the use and distribution of digital multimedia data. Digital multimedia information has many advantages compared with analog one. In contrast, it is also easy to realize unauthorized copying and redistribution. Therefore, the copyright protection of digital multimedia content has been become an improtant research area. The most promising solution for the copyright protection seems to be digital watermarking technology [1], in which robustness, security and inaudibility are three fundamental requirements [2]. Unfortunately, few watermarking methods have satisfying characteristics for these three demands. By now, SS (spread spectrum) technique has been popularly used in robust audio watermarking applications [3]. In most cases, the algorithms in watermarking methods employ the binary or real-value PN sequences to modulate the watermarking information, and detect the watermarking information using a blind correlation $[3,4]$. As one is known that, in communication system, a quadri-phase SS sequence has shown a better correlation than binary or real-value one [5]. In this letter, we propose a novel audio watermarking algorithm using quadri-phase SS sequence, which generated by unpredicted chaotic map [6]. It improves the robustness and security for the watermarking application, and the detection is a blind SS watermarking scheme in the transform domain. Furthermore for the inaudibility purpose, we applied the psychoacoustic masking model [4] to control the watermarking strength. The estimation of comparability between different scale wavelet subbands is used to enhance the reliability of the detection result. The experimental results show that property of the proposed scheme is significantly improved.

\section{Watermark embedding algorithm}

Quadri-phase sequence plays an important role in our algorithm. In this paper, two unpredicted chaotic spreading sequences [6] are considered as seed sequences used for the generation of quadri-phase sequence. The unpredicted chaotic map is denoted in formula (1), and the generated sequence is of a strongly chaotic nature, it can ensure the watermarking security.

$$
\begin{gathered}
x_{n+1}=\sin ^{2}\left(Z \times \arcsin \left(\sqrt{x_{n}}\right)\right), \quad 0 \leq x_{n} \leq 1, \\
n=0,1,2, \ldots, Z \text { is a real number }
\end{gathered}
$$

In [6], it is proved that the probability density of $x_{n}$ is symmetry, the symmetric center point is $x=0.5$. Therefore, we can construct quadri-phase sequences with zero mean as formula (2):

$$
Q_{i}=\left\{\begin{array}{ll}
+1 & 0.5<x^{(1)}<1,0.5<x_{i}^{(2)}<1 \\
+j & 0<x^{(1)}{ }_{i}<0.5,0.5<x^{(2)}{ }_{i}<1 \\
-1 & 0.5<x^{(1)}{ }_{i}<1,0<x^{(2)}{ }_{i}<0.5 \\
-j & 0<x^{(1)}<0.5,0<x^{(2)}{ }_{i}<0.5
\end{array} \quad i=1,2, \ldots, N\right.
$$

Here, $x^{(1)}, x^{(2)}$ are two different unpredicted chaotic spreading sequences with the same length, and $Q$ denotes the quadri-phase sequence. In watermark embedding process, the copyright information is modulated by $Q$. Let $w$ denotes one sequence in which single-bit copyright information is carried. In order to guarantee the inaudibility of the watermark signal, we use frequency psychoacoustic masking model to control the strength of $w$ [4].

The watermark signal of the proposed algorithm is embedded in the wavelet transform domain of the original audio signal. The tree structure of wavelet analysis is showed in Fig. 1: Here, $S$ is the original audio signal. $a^{1}, d^{1}, a^{2}, d^{2} \ldots$ are the wavelet subbands of $S$. In this letter, we select $a^{3}, d^{3}$ for embedding the watermarking information. The embedding process can be represented using the formulas (3) and (4):

$$
\begin{aligned}
& M a^{3}{ }_{w}=a^{3}{ }_{w}+\beta_{1} \times \operatorname{Re}(w) \\
& M d^{3}{ }_{w}=d^{3}{ }_{w}+\beta_{2} \times \operatorname{Im}(w)
\end{aligned}
$$

The $a^{3}{ }_{w}$ and $d^{3}{ }_{w}$ are the corresponding coefficients extracted from $a^{3}$ and $d^{3}$ respectively. $\beta_{1}$ and $\beta_{2}$ are watermarking power factors which be decided by the perceptual masking thresholds. In order to avoid the real part of quadri-phase watermarking information overlapping the imaginary part in the wavelet synthesis, the samples extracted from $a^{3}$ and $d^{3}$ respectively should have different position indexes, that is: $a^{3}{ }_{w}=a^{3}(p), d^{3}{ }_{w}=d^{3}(q), p \cap q=\Phi$. In this way, the watermarked samples can be first construct from $a^{3}{ }_{w}$ and $d^{3}{ }_{w}$. And then, perform the wavelet synthesis on $M a^{3}, M d^{3}$ and other retrieval wavelet subbands, we can reconstruct the watermarked audio signal.

\section{Watermark detection}

The first step in the watermark detection is as same as the embedding process. Here, let $S^{\prime}$ denote a signal to be detected, the signal is first decomposed by wavelet analysis, and then the corresponding coefficients are selected from the subbands 


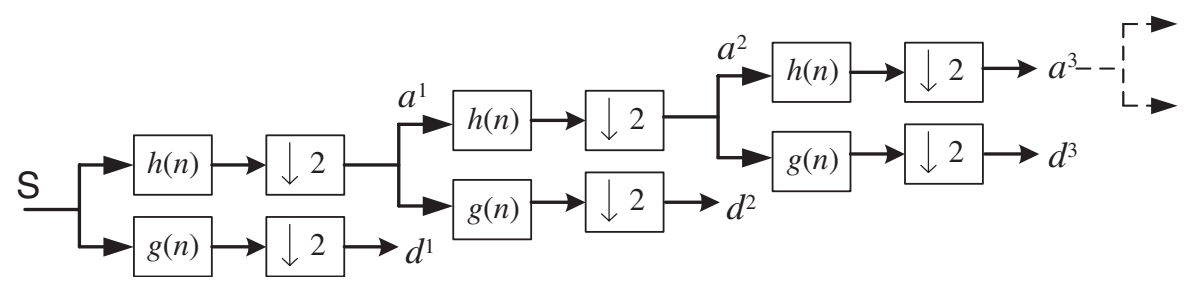

Fig. 1 Tree of filters for wavelet analysis.

$a^{3^{\prime}}$ and $d^{3^{\prime}}$. For the purpose of reducing the influence on correlation result caused by original audio signal, we propose a novel estimation to enhance the correlation performance.

$$
\begin{aligned}
& D a^{3^{\prime}}=\frac{a^{3^{\prime}}}{\left\|a^{3^{\prime}}\right\|}-\frac{T a^{4^{\prime}}}{\left\|T a^{4^{\prime}}\right\|} \\
& D d^{3^{\prime}}=\frac{d^{3^{\prime}}}{\left\|d^{3^{\prime}}\right\|}-\frac{T d^{4^{\prime}}}{\left\|T d^{4^{\prime}}\right\|}
\end{aligned}
$$

In formulas (5) and (6), $T a^{4^{\prime}}$ and $T d^{4^{\prime}}$ are interpolated results of $a^{4^{\prime}}$ and $d^{4^{\prime}}$, and they have same length as $a^{3^{\prime}}$ and $d^{3^{\prime}}$. The generalized odd correlation between the residual quadri-phase sequence $D a^{3^{\prime}}+j^{*} D d^{3^{\prime}}$ and $Q$ is defined as the output of watermark detection. Let $D_{w}$ denote a $N$-point length residual quadri-phase sequence. The generalized odd correlation between $D_{w}$ and $Q$ is calculated as the following formulas:

$$
\begin{aligned}
& D_{w}=D a^{3^{\prime}}{ }_{w}+j^{*} D d^{3^{\prime}}{ }_{w} \\
& r_{w}(\tau)=\left\{\begin{array}{l}
\sum_{i=0}^{N-x-1}\left(D_{w}(i) \times Q^{*}((i+\tau) \bmod N)\right) / N, 0 \leq \tau \leq N-1 \\
\sum_{i=0}^{N-1+x}\left(D_{w}((i-\tau) \bmod N) \times Q^{*}(i)\right) / N,-(N-1) \leq \tau \leq 0
\end{array}\right. \\
& R_{w}(\tau)=\left|r_{w}(\tau)-r_{w}(\tau-L)\right|
\end{aligned}
$$

where $Q^{*}$ is the complex conjugate sequence of $Q$. According to the values of $R_{w}$, it could be determined obviously whether the $D_{w}$ has embedded watermarking information or not.

\section{Experimental results}

The algorithm has been applied to a set of audio samples, such as music, speech, and other mixed sound. As an example, a 16 bits' music audio sampled at $44.1 \mathrm{kHz}$ has been used to test this algorithm. In the subjective quality test conducted among 8 listeners, the watermarked signal is undistinguishable from the original audio signal. The response results of the watermarking detector in the noiseless watermarking channel are shown in Fig. 2. The experimental results have been compared with the results of a quadric-phase watermarking scheme. The left graph of Fig. 2 is the detector response of the proposed algorithm, and the right graph is the detector response of a quadric-phase algorithm. It is obviously that our method has an excellent correlation peak to side-lode ratio comparing to the result of binary watermarking scheme. In order to validate the robustness of this algorithm, the watermarked signal was attacked using noise addition, MPEG encoding and low-pass filtering. The detection results after these attacks are shown in Fig. 3. Where, signal to noise ratio (SNR) of the signal added uniform noise is $20.36 \mathrm{~dB}$, the cutoff frequency of the low-pass filter is fixed at $4 \mathrm{kHz}$ and the bit rate after MPEG encoding is $64 \mathrm{kbps}$. Graphs (a), (b) and
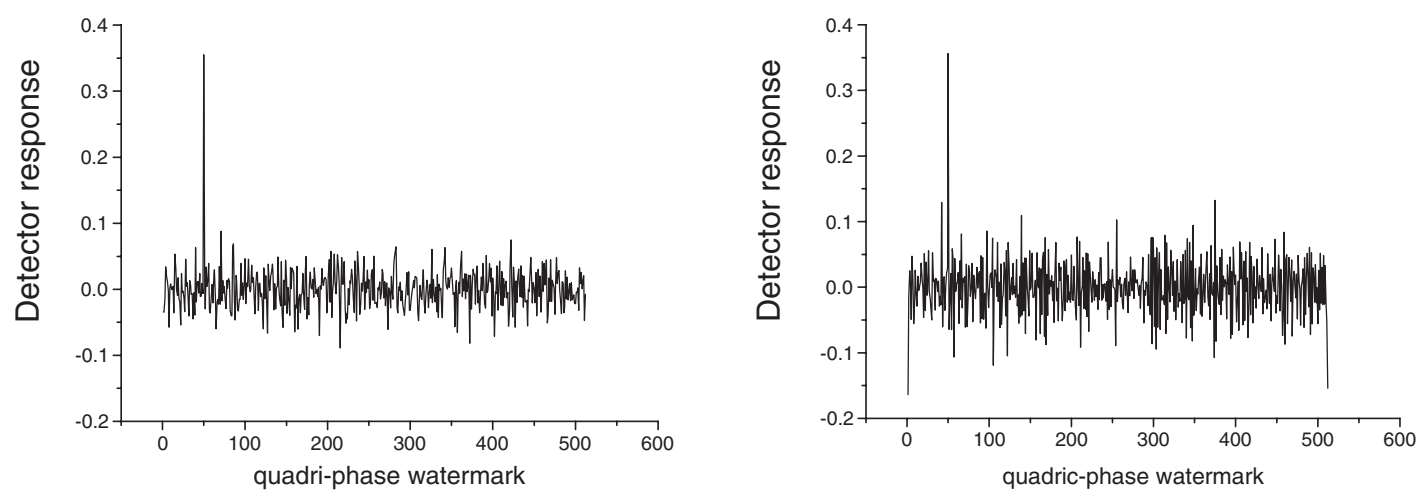

Fig. 2 The detection results of noiseless watermarking channel. 


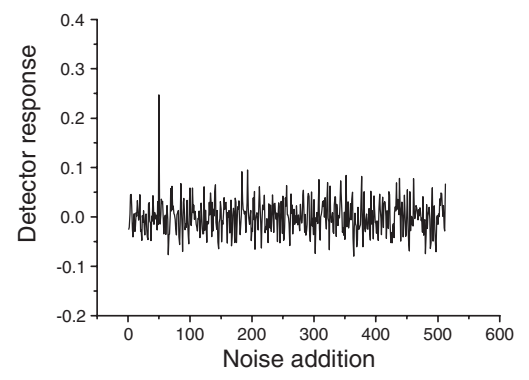

(a)

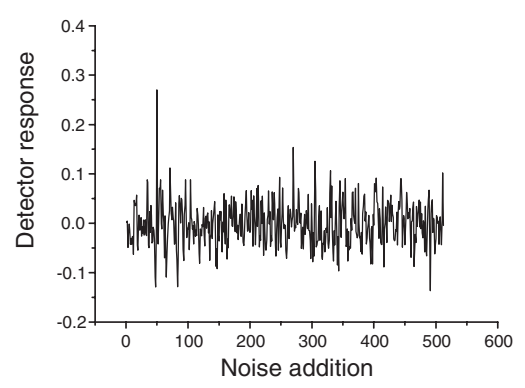

(d)

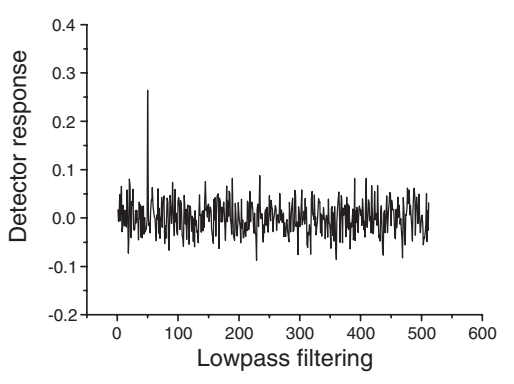

(b)

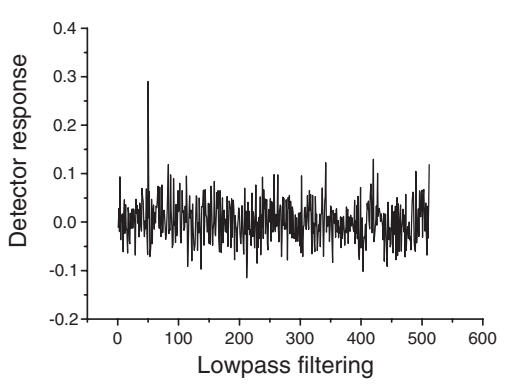

(e)

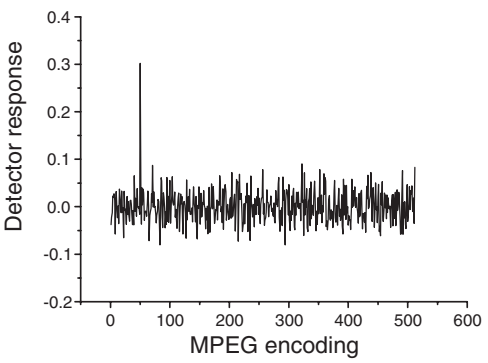

(c)

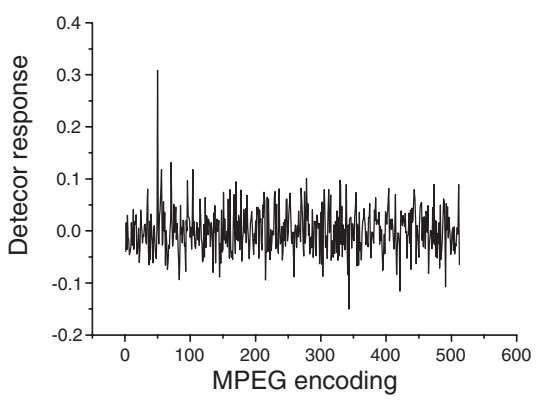

(f)

Fig. 3 The detection results of attacked watermarking channel.

(c) of Fig. 3 are detection responses of the quadri-phase watermarks after attacks of noise addition, low-pass filtering and MPEG encoding respectively, others are detection results of audio sample watermarked by quadric-phase sequences with the same length after attacked. Comparing the detection values achieved with the two different watermarking schemes (a) compares with (d), (b) compares with (e) and (c) compares with (f) respectively), we can see that the correlation peaks of both schemes are approximately equivalent but the sidelobe suppressions of quadri-phase watermark are much better. It is obviously to see that the attack operations may cause the degradation of correlation value in a certain extent, however the proposed method could achieve better detection properties.

\section{Conclusions}

In this paper, we propose a novel quadri-phase audio watermark. Different from traditional watermarking algorithms, the watermark information is modulated by quadriphase unpredicted chaotic spreading sequence and then embedded in the subbands of wavelet transform domain. The psychoacoustic masking model is used to guarantee the inaudibility of watermark. In watermark detection, we employ a novel estimation to improve the performance of the watermarking detector. Experiment results show that this method has a good robustness against several attacks.

\section{References}

[1] G. Voyatzis and I. Pitas, "The use of watermarks in the protection of digital multimedia products," Proc. IEEE, 87, 1197-1207 (1999).

[2] W. Bender, D. Gruhl and N. Morimoto, "Techniques for data hiding," IBM Syst. J., 35, 313-336 (1996).

[3] I. J. Cox, J. Kilian, F. T. Leighton and T. Shamoon, "Secure spread spectrum watermarking for multimedia," IEEE Trans. Image Process., 6, 1673-1687 (1997).

[4] M. D. Swanson, B. Zhu, A. H. Tewfik and L. Boney, "Robust audio watermarking using perceptual masking," Signal Process., 66, 337-355 (1998).

[5] D. He, C. He, L. Jiang and G. Hu, "Correlation characteristic of binary-phase and quadric-phase ICMIC spreading sequences," J. Chin. Inst. Commun., 22, 13-19 (2001).

[6] J. A. Gonzalez and R. Pino, "Chaotic and stochastic functions," Physica A, 276, 425-440 (2000). 